\title{
TTVs study in southern stars
}

\author{
Romina Petrucci ${ }^{1}$, Emiliano Jofré ${ }^{2}$, Martín Schwartz $^{1}$, \\ Andrea Buccino ${ }^{1}$ and Pablo Mauas ${ }^{1}$ \\ ${ }^{1}$ Instituto de Astronomía y Física del Espacio (IAFE), Buenos Aires, Argentina \\ email: romina@iafe.uba.ar, mschwartz71@gmail.com, abuccino@iafe.uba.ar, \\ pablo@iafe.uba.ar \\ ${ }^{2}$ Observatorio Astronómico de Córdoba (OAC), Córdoba, Argentina \\ email: emiliano@mail.oac.uncor.edu
}

\begin{abstract}
In this contribution we present 4 complete planetary transits observed with the 40cm telescope "Horacio Ghielmetti" located in San Juan(Argentina). These objects correspond to a continuous photometric monitoring program of Southern planet host-stars that we are carrying out since mid-2011. The goal of this project is to detect additional planetary mass objects around stars with known transiting-planets through Transit Timing Variations (TTVs). For all 4 transits the depth and duration are in good agreement with the values published in the discovery papers.
\end{abstract}

Keywords. extrasolar planets, transiting-planets, TTVs

\section{Introduction}

So far, over 690 extrasolar planets has been detected by different techniques; radial velocities and transits methods have provided the majority of detections. Period, orbital eccentricity and minimum mass of the planet can be determined by the first technique, meanwhile period, inclination and relative radii of the planet can be obtained by the second one. Therefore, it is possible to calculate the planetary density combining spectroscopic and photometric observations. The disadvantage is that spectroscopic measurements are limited by the star magnitude.

On the other hand, there is another exoplanet detection technique, currently very popular, based on the fact that the time interval between successive transits of an unperturbed planet is always the same. However the presence of another planetary mass body in the system can produce variations of the transiting-planet period due to their mutual gravitational interaction. These transit timing variations (TTVs) depend on the mass of the additional planet, and in some cases terrestrial-mass planets will produce a measurable effect. Furthermore, systems in which two planets transit their star, the masses and radii of each planet can be determined without spectroscopic measurements (Holman \& Murray 2005). Therefore, it is possible to compute densities even for faint, low-mass stars. This is one of the aspects that makes TTVs a very important detection technique.

\section{The Telescope}

The observations of the 4 transits were made remotely with the $40-\mathrm{cm}$ telescope "Horacio Ghielmetti" located on "Cerro Burek" at CASLEO (San Juan, Argentina). This is a MEADE-RCX 400 telescope, $f / 8$, with a Ritchey-Chretien advanced optical system, equipped with an APOGEE ALTA U16M camera and UBVRI filters. The image on the CCD covers an area of $49^{\prime} \times 49^{\prime}$. 


\section{First results}

Figure 1 (a to d) shows the light-curves corresponding to the exoplanet host-stars: WASP-28, WASP-44, GJ-1214 and WASP-4. All the observations were taken in the clear filter to improve the temporal resolution, and the exposure times were chosen so that the comparison stars and the exoplanet host-stars do not saturate the CCD camera. We corrected the images for bias and dark with standard tasks of the IRAF package program. After calibration, we made aperture photometry for determining instrumental magnitudes using the "DAOPHOT" package. For each star in the stellar field the adopted aperture size was that for which the star magnitude was stable in 0.001 magnitudes (Howell 1989), the annulus was 5 pixels from the aperture, and the dannulus was 5 pixels. Then, we made a first order extinction correction, taking as a reference non variable stars with similar color to the target star. We fitted each resulting light-curve with an iterative method described in Poddany et al. 2010.

In the next sections we present more characteristics of each particular case:

\subsection{WASP-28}

It is an object of spectral type $\mathrm{G}, \mathrm{V}=12$, with a "hot Jupiter" planet. It has a $M_{P}=$ $0.91 M_{\text {jup }}$ and $\mathrm{P}=3.40$ days. The transit shown in the figure $1 \mathrm{a}$ ) was observed on the night of August 27, 2011. The exposure time was 120 seconds and we used 2 reference stars. The data dispersion is $\sigma=4.3 \mathrm{mmag}$ and the fit corresponds to a depth of $19.2 \pm$ $1.2 \mathrm{mmag}$ and a duration of $199.4 \pm 4.6 \mathrm{~min}$.

\subsection{WASP-44}

This is a $\mathrm{V}=12.9$, G8V star, that posses a planet $\left(M_{P}=0.89 M_{\text {jup }}\right)$ with a 2.42 days period (Anderson et al. 2011). Figure 1b) shows the transit observed on the night of August 28, 2011. We used an exposure time of 150 seconds and 3 reference stars. The dispersion of the points is $\sigma=4.7 \mathrm{mmag}$ and the fit corresponds to a depth of $18.45 \pm$ $1.5 \mathrm{mmag}$ and a duration of $148.3 \pm 5.6 \mathrm{~min}$.

\subsection{GJ-1214}

It is a $\mathrm{M}$ star with $\mathrm{V}=14.67$ that hosts a super-Earth planet $\left(M_{P}=6.6 M_{\text {earth }}\right.$ and $R_{P}=2.7 R_{\text {earth }}$ ) (Berta et al. 2011). The transit shown in the figure 1c) was observed on the night of July 2, 2011. We adopted an exposure time of 150 seconds and used 7 reference stars. As can be seen, in the points corresponding to the "out-of-transit" after the transit, appears a decrease in magnitudes that could not be removed. This is probably due to an instrumental error or due to the fact that the colors of the comparison stars differ significantly from the color of GJ-1214. The dispersion is $\sigma=3.5 \mathrm{mmag}$ and the fit corresponds to a depth of $16.6 \pm 2.9 \mathrm{mmag}$ and a duration of $56.5 \pm 10.5 \mathrm{~min}$.

\subsection{WASP-4}

This star has a magnitude of $\mathrm{V}=12.5$ and hosts a planet of $M_{P}=1.21 M_{\text {jup }}$ (Gillon et al. 2009). The transit shown in the figure 1d) was observed on the night of October 17, 2011. The exposure time was 25 seconds and we used 5 reference stars. The data dispersion is $\sigma=6.6 \mathrm{mmag}$ and the fit corresponds to a depth of $31.2 \pm 1 \mathrm{mmag}$ and a duration of $126.9 \pm 2.1 \mathrm{~min}$. 

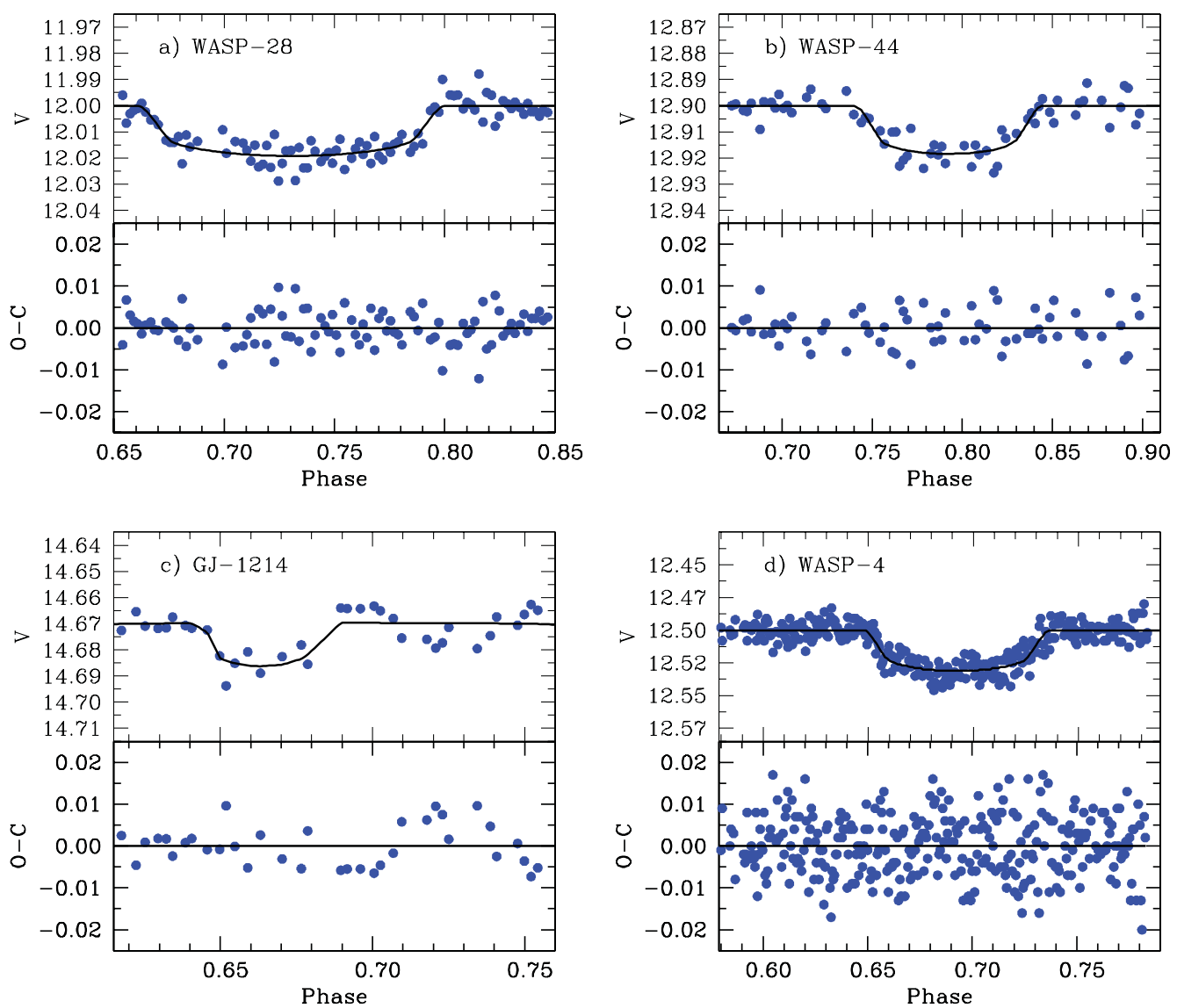

Figure 1. Light-curves and residuals for a) WASP-28, b) WASP-44, c) GJ-1214 and d) WASP-4. In the light-curves the blue dots correspond to the observations and the continuos black line represents the best fit to the data.

\section{Conclusion and future steps}

In all 4 cases the values of depth and duration of the planetary transits obtained in this work agree with those published in the discovery papers. Our next step is to investigate and test different algorithms to remove systematic effects and decrease data dispersion. In future works, we will use the Mandel and Algol code (Mandel \& Algol 2002) for fitting the light-curves and we will improve the precision of minimum time measurements. In the following months we will also continue with the planetary transit observations remotely and will begin with the TTVs analysis.

\section{Acknowledgments}

We would like to thank the CASLEO staff for helping to maintain the telescope, to Pablo Perna and Matías Pereyra for providing software support and, finally, Romina Petrucci and Pablo Mauas thank IAU for providing financial support to attend this Symposium. 


\section{References}

Anderson, D. R., Collier Cameron, A., \& Gillon, M., et al. 2011, MNRAS in press.

Berta, Z. K., Charbonneau, D., \& Bean, J., et al. 2011, ApJ, 736, 12

Gillon, M., Smalley, B., \& Hebb, L., et al. 2009, AछA, 496, 259

Holman, M. J. \& Murray, N. W. 2005, Science, 307, 1288

Howell, S. B. 1989, PASP, 101, 616

Poddany, S., Brat, L., \& Pejcha, O. 2010, New Astron., 15, 297 